\title{
Excimer laser micromachining of oblique microchannels on thin metal films using square laser spot
}

\author{
SYED NADEEM AKHTAR ${ }^{1, *}$, SHASHANK SHARMA ${ }^{1}$, S ANANTHA RAMAKRISHNA ${ }^{2}$ and \\ J RAMKUMAR $^{1}$
}

${ }^{1}$ Department of Mechanical Engineering, Indian Institute of Technology Kanpur, Kanpur 208016, India

${ }^{2}$ Department of Physics, Indian Institute of Technology Kanpur, Kanpur 208016, India

e-mail: syed.nadeem.akhtar@gmail.com

MS received 23 January 2015; revised 24 November 2015; accepted 18 January 2016

\begin{abstract}
Excimer laser micromachining of thin metal films with a sacrificial polymer coating is a novel technique that produces features with smooth edges. Using this technique, oblique microchannels are fabricated by workpiece dragging and using a square laser spot, where the axis of traverse of the workpiece is not parallel to the edges of the square laser spot. The microchannels have serrated edges that are particular to the shape of the mask producing the spot. The edge roughness of the channels, machined with a square laser spot of side 100 $\mu \mathrm{m}$, is found to be most affected by the fluence-spot overlap interaction, and the channel width by spot-overlap and the angle of tilt of the traversed path. Polymer coated metal films underwent close to ideal machining, aided by the clamping action of the polymer layer. Through this technique of machining post polymer coating, the edge roughnesses of the microchannels have been curtailed to less than $10 \mu \mathrm{m}$, and channel widths to $150 \mu \mathrm{m}$. This technique may be used in fabrication of oblique and circular patterns using excimer laser micromachining with rectangular and square laser spots.
\end{abstract}

Keywords. Excimer laser micromachining; polymer coated metal film; film machining.

\section{Introduction}

Micro patterning of metallic thin films finds applications in manufacturing of MEMS devices, electronic circuit boards, miniaturized microwave circuits, and photonic devices. Such micro-patterned metallic films can offer exceptional electromagnetic and optical properties [1-3] for use in, for example, photovoltaic technologies, beam shaping and laser applications [3-6]. Laser machining [7] of thin metal films has long been an area of research due to its use in device fabrication technologies. Excimer lasers, in particular, offer several advantages such as high laser-material coupling due to low reflectivity of metals in the UV wavelength range, sub-micron resolution due to small wavelength, minimal heat affected zone and high beam energy due to short pulse duration and high cross-sectional uniformity [8].

The mechanisms of laser ablation of bulk metals and thin metal films are different from each other. Whereas bulk metal ablates primarily through heating and vaporization, thin metal films have been observed to ablate much below the fusion temperature of the metal and the bulk ablation threshold [8]. As an example, thin films of tungsten have

*For correspondence been observed to ablate in solid pieces flying directly away from the substrate, at low fluences [9]. Molten and vaporized material was found to be ejected only at very high fluences. Hence the metal film undergoing ablation gets pushed out from the interface due to an ejection force. Because of the thermo-mechanical nature of thin film ablation, the irradiated portion carries with itself the nonirradiated portion from its surroundings. This peeling of the surrounding film [10] causes excessive lateral ablation and distortion in feature shape. The adhesion of the metal films to the (glass) substrates dictates, to a large extent, the quality of features formed upon ablation. The oxygenaffinity of the metal and the thickness of the oxide layer at the metal-substrate interface determine the adhesion strength of the film [11].

It has been demonstrated in one of our earlier works [12] that excimer laser micromachining of a thin metal film can be made precise by coating the metal film with a sacrificial thin film of a polymer, whereby the polymer film protects the metal film from tearing during the ablation process. Thus, it is a better (simpler and quicker) alternative to various other techniques currently being used to fabricate the above-mentioned devices. The ability to machine microfeatures (straight channels) at lengthscales ranging from 100 micrometers to a single micrometer on thin 
aluminum, chromium and gold films coated on borosilicate glass has been demonstrated. However, fabricating devices, such as the ones mentioned in the preceding paragraphs, often require fabricating curved features, such as circular pads, and interconnects. In order to achieve sharp corners, a square or rectangular mask is often used to produce a projected and demagnified laser spot with sharp features. This, however, causes difficulty in making oblique or curved features where the axis of traverse of the laser spot does not align with the edges of the square/rectangular spot. The produced line or feature has a rough or serrated edge due to successive pulses falling on displaced areas. In such a case, the factors that determine the quality of the feature are the spot overlap and the angle of tilt in addition to the laser fluence. Spot overlap is a measure of the area that is common to two consecutive laser spots. The angle of tilt is a measure of the lateral offset of successive laser spots from a horizontal or a vertical line. Using these three factors, experiments are conducted to fabricate oblique microchannels. The microfeatures are characterized for their edge roughness and channel width. The polymer coated metal films are shown to be machined with sharp boundaries in comparison with uncoated metal films.

\section{Experimental setup and procedure}

The schematic diagram of the experimental setup is shown in figure 1 . The pulsed $\mathrm{KrF}$ excimer laser with a wavelength of $248 \mathrm{~nm}$ is used. $200( \pm 5) \mathrm{nm}$ thick films of aluminum and chromium are deposited by thermal evaporation on cleaned borosilicate glass substrates. On some of the metal films, $1 \mu \mathrm{m}$ thick film of PVA (polyvinyl alcohol, hereafter also referred to as polymer, unless specified otherwise) is spin coated. Using a mask projection method [13], a $100 \mu \mathrm{m} \times 100 \mu \mathrm{m}$ square spot with uniform irradiance across the spot is obtained on the workpiece. Assuming the sides of the square spot are aligned with the $X$ and $Y$ axes (as shown in figure 1), scanning along $X$ (or $Y$ ) axis produces straight channels, whereas scanning along any other direction produces oblique channels as defined in this

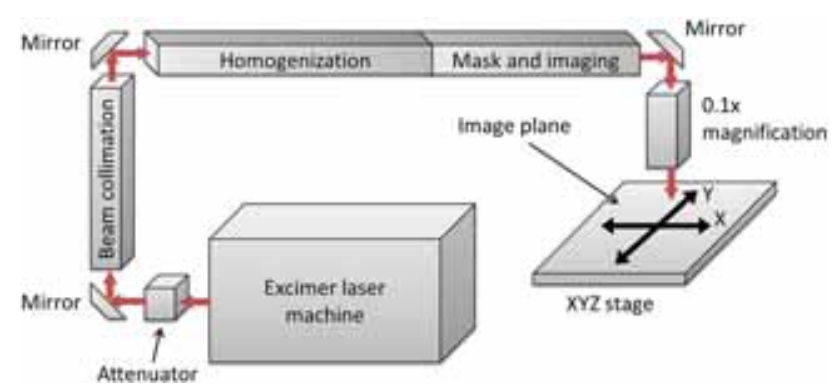

Figure 1. The schematic diagram of the excimer laser micromachining setup, showing the beam delivery section, and the image plane of the mask, at which the workpiece is kept.
Table 1. Levels of input parameters varied during the experiment.

\begin{tabular}{lrrrrr}
\hline Factors & $-\alpha$ & \multicolumn{1}{c}{-1} & \multicolumn{1}{c}{0} & \multicolumn{1}{c}{+1} & $+\alpha$ \\
\hline Fluence $\left(\mathrm{J} / \mathrm{cm}^{2}\right)$ & 0.6 & 0.9 & 1.3 & 1.7 & 2.0 \\
Overlap (\%) & 6.3 & 20.0 & 40.0 & 60.0 & 73.6 \\
Angle (degrees) & 1.1 & 10.0 & 23.0 & 36.0 & 44.9 \\
\hline
\end{tabular}

study. The experiments are designed using the response surface methodology [14], and aim to obtain a qualitative assessment of the effects of sacrificial polymer layer on edge roughnesses and widths of the channels fabricated, and the effects of interaction between the input variables. Three machining parameters are considered during machining, namely, fluence, spot overlap (hereafter referred to as overlap) and angle of tilt (hereafter referred to as angle). The high and low values of fluence and spot overlap have been determined using preliminary experiments, whereas those of angle of tilt were determined in a manner that captures all possible angles. The other values have been determined using the central composite design, with $\alpha$ set at 1.682. The values are tabulated in table 1 .

The machined features are characterized for the edge roughness and channel width. The procedure of image acquisition and processing is discussed in detail elsewhere [15], and is briefly described here. The channels are encapsulated within the dark background by editing the images. The edges, and hence the objects (channels) within the image, are detected using Canny algorithm, after converting the image to gray scale and then to binary. The gaps in the image are filled by dilating the image, and the boundaries of the channel are outlined (edge detection). The coordinates of each edge are stored one by one. After the channel edges are extracted, straight lines are fitted to both the edges of the channel using the least sum of squares fit. The perpendicular distance $(d)$ of the points on the edge from the fitted line is used to calculate the root mean square of the distance (also called $R_{r m s}$ or $R_{q}$ ), which is the edge roughness.

Channel width is defined as the distance between two straight lines, fitted to the outermost edges of the channel. The edge roughness and channel width are measured with an accuracy of a couple of pixels, where one pixel corresponds to two micrometers. A third-order regression model was fitted to the experimental data to obtain an empirical relation between the aforementioned input parameters. Separate models for edge roughness and channel width were obtained for each case. To scrutinize the correction of fit and to eliminate the insignificant terms from the fitted model, analysis of variance was used. Equations were fitted to the observed data, with individual factors up to third order, and interaction terms with individual factors up to second order. The confidence interval used was 95\%, and all model terms were significant, having low p-values (less than 0.01). 


\section{Results and discussion}

The thermal diffusion lengths [calculated as $2 \sqrt{ }\left(\kappa t_{p} / \rho C_{p}\right)$, where $\kappa, t_{p}, \rho$ and $C_{p}$ represent the thermal conductivity, pulse duration, density and specific heat capacity, respectively] for aluminum and chromium, for a $20 \mathrm{~ns}$ laser pulse, are $2.8 \mu \mathrm{m}$ and $1.5 \mu \mathrm{m}$ respectively. The optical skin depths are $15 \mathrm{~nm}$ and $11 \mathrm{~nm}$ respectively in aluminum and chromium. Clearly, the thickness of the metal films $(100 \mathrm{~nm})$ was almost an order of magnitude larger than the optical penetration depth and an order of magnitude smaller than the thermal diffusion depth. It is inferred from the above that there is little thermal gradient across the thickness of the film, and also that the ablation threshold of the film is about an order of magnitude smaller than the bulk ablation threshold [16]. The optical micrographs of some straight and oblique channels fabricated during the study are shown in figure 2 . The models fitted to the observations of edge roughness and channel width, for both plain and PVA coated aluminum and chromium films, are mentioned in table 2.

\subsection{Ideal oblique microchannel}

The schematic diagrams of channels tilted at various angles and with varying spot overlaps are shown in figure 3. One of the first observations from the schematic diagrams shows that with variation in fluence, the diagram does not change. Hence, the edge roughness and channel width of an ideal channel were not affected by fluence. Channels made at same spot overlap (73.6\%) are shown in figure $3 \mathrm{a}$ and $3 \mathrm{c}$. Upon comparison of the two, it is observed that the channel machined at greater angle had a higher edge roughness and higher width. Hence edge roughness and width increased with the angle of tilt. Similarly, comparing channels in figure $3 b$ and $3 c$, both of which were machined at the same angle of tilt, it can be inferred that the edge roughness

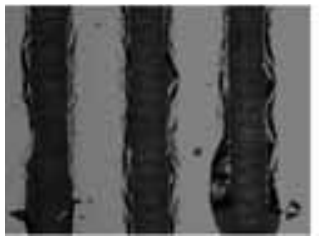

(a)

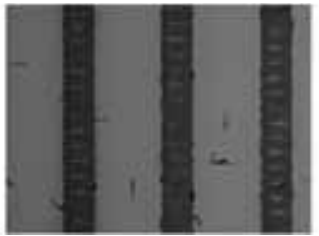

(b)

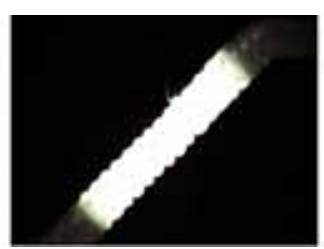

(c)

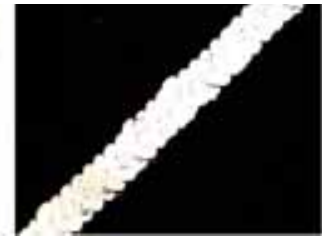

(d)

Figure 2. Optical micrographs of channels, machined with a $100 \mu \mathrm{m}$ wide laser spot at $1.3 \mathrm{~J} / \mathrm{cm}^{2}$ and $40 \%$ spot overlap, in aluminum and chromium films, shown for the purpose of comparison. The two images on the left are those of straight channels, and the two on the right are those of oblique channels (machined at $44.9^{\circ}$ ). (a) Aluminum film, (b) Chromium film, (c) Aluminum film, and (d) Chromium film.

Table 2. Details of the third order regression models fitted to the experimentally observed values of edge roughness and channel width of tilted channels machined in aluminum and chromium, both without and with PVA coating.

\begin{tabular}{|c|c|c|c|c|c|c|c|c|}
\hline \multirow[b]{2}{*}{ Model terms } & \multicolumn{4}{|c|}{ Edge roughness } & \multicolumn{4}{|c|}{ Channel width } \\
\hline & $\mathrm{Al}$ & Al-PVA & $\mathrm{Cr}$ & Cr-PVA & $\mathrm{Al}$ & Al-PVA & $\mathrm{Cr}$ & Cr-PVA \\
\hline Constant & 3.39 & 4.75 & 5.95 & 5.85 & 170.19 & 142.11 & 176.35 & 126.93 \\
\hline A - Fluence & 1.29 & -0.47 & & -0.26 & 10.58 & 2.21 & 7.84 & 4.21 \\
\hline B - Spot overlap & & -3.07 & & -1.82 & & 7.6 & & 5.24 \\
\hline $\mathrm{C}$ - Angle of tilt & 0.56 & 1.7 & 0.48 & 2.29 & 6.35 & 5.23 & & 7.85 \\
\hline $\mathrm{AB}$ & & 0.29 & -0.95 & & -1.9 & -2.55 & & \\
\hline $\mathrm{AC}$ & & -0.36 & -0.45 & & & -3.05 & & \\
\hline $\mathrm{BC}$ & -0.36 & -1.01 & -0.62 & -0.97 & 1.76 & 5.62 & & 2.52 \\
\hline$A^{2}$ & 1 & 0.21 & & & & & & \\
\hline $\mathrm{B}^{2}$ & & -0.74 & & & & 1.66 & & \\
\hline$C^{2}$ & & -0.21 & & -1 & & -1.02 & -4.56 & \\
\hline$A^{2} B$ & -1.38 & 1.64 & & & -2.69 & & & \\
\hline $\mathrm{AB}^{2}$ & -1.28 & & & & & & & \\
\hline $\mathrm{A}^{2} \mathrm{C}$ & 0.51 & & & & & 3.58 & & \\
\hline $\mathrm{ABC}$ & & 0.23 & & & 2.21 & -2.75 & & \\
\hline Model F value & 24.26 & 123.35 & 10.64 & 127.37 & 64.41 & 49.18 & 7.25 & 21.25 \\
\hline
\end{tabular}




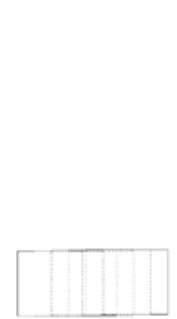

(a)

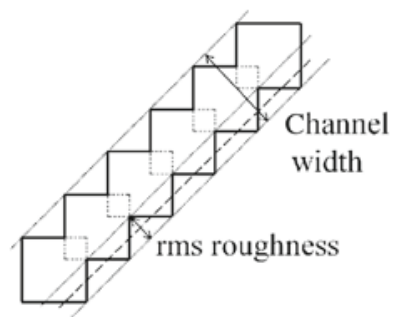

(b)

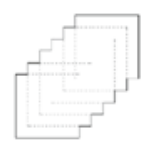

(c)
Figure 3. The schematic diagrams of oblique microchannels fabricated at different angles and spot overlaps. The edge roughness increases with angle and decreases with spot overlap. The channel width only depends on the angle of tilt, and increases with it. (a) Angle $1.1^{\circ}$, spot overlap $73.6 \%$, (b) Angle $44.9^{\circ}$, spot overlap $6.3 \%$, and (c) Angle $44.9^{\circ}$, spot overlap $73.6 \%$.

increased with decrease in spot overlap, and the channel width remained independent of the spot overlap.

\subsection{Aluminum film machining (without and with polymer coating)}

A thorough study on the direct ablation of aluminum film while making straight channels with $100 \mu \mathrm{m}$ laser spots, with their axes parallel to the edges of the square spot, has been presented in the work of [15]. Fluence was found to have more than $80 \%$ contribution towards determining the edge roughness and channel width. The edge roughness and channel width had a little dependence on the spot overlap for straight microchannels. Here, the feature quality of oblique microchannels is discussed. The variations of edge roughness and channel width with fluence, angle and overlap, for channels machined with $100 \mu \mathrm{m}$ wide laser spots are shown in figure 4 . As seen in the figures, the ideal roughness, denoted by the solid line, is independent of the fluence, and it increased with angle and decreases with overlap. This has been discussed in the preceding subsection. For the case of the plain aluminum film, denoted by the dotted lines, it is observed from figure $4 \mathrm{a}$ that roughness remained fairly unaffected by fluence. Considering the effect of fluence alone, it had a $38 \%$ contribution to the edge roughness, and the roughness actually increased with fluence. This was due to development of larger ejection pressures at higher fluences that also got dissipated sideways and caused ejection of both radiated and the surrounding irradiated portions of the metal film. Aluminum is known to undergo large lateral ablation, with lateral overcut of the order of the intended width [12]. This is a result of poor adhesion of aluminum to glass [17], softness, low melting point of aluminum and outgassing from the glass substrate. The combination of these three factors makes aluminum thin films highly prone to peeling/delamination and getting scratched. Aluminum films are much more uniform with large grain sizes. Absence of grain boundaries in aluminum films allows large pieces to be ejected from the surrounding regions near the grain boundary, generating a rougher profile. However, interactive effects of fluence and overlap, with $51 \%$ contribution, caused the roughness to decrease. Hence, the two factors, fluence as an independent factor, and fluence-overlap interaction, had opposing effects on the roughness, tending to negate the effects of each other. This is evident from figure $5 \mathrm{a}$, where both the above-mentioned factors are found to have almost equal contributions to the edge roughness, albeit in opposite senses.

Edge roughness, ideally and actually, decreased with increasing overlap, as shown in figure $4 \mathrm{c}$. (This is unlike the ablation of straight channels, where overlap has little role to play, even in interaction with other factors.) At high overlap, the successive pulses see a little unablated material. Hence additional ablation by a pulse was small, leading to lower roughness. However, it is to be noted that overlap did not affect the roughness independently (refer figure 5a), rather only at small fluence. High fluence pulses caused such excessive ablation that even at small overlaps, successive pulses saw little unablated material. Edge roughness increased with angle, as shown in figure $4 \mathrm{~b}$. Such a response is expected, as at higher angles, successive pulses fall at higher offsets from the preceding ones. It can be observed from figure 3 that at higher angles (or offset), a pulse sees unablated material on all four sides. This leads to excessive ablation and hence increase in roughness. However, the contribution of the angle towards the edge roughness was significantly lower than those of fluence and overlap, as can be seen from figure $5 \mathrm{a}$.

The ideal width, as already discussed, does not depend on the fluence or overlap. However, the width of the channel was observed to increase with fluence (dotted line) as shown in figure $4 \mathrm{~d}$. This is attributable to the excessive lateral ablation in case of machining aluminum film directly, an observation already discussed in the preceding paragraphs. Understandably so, with $60 \%$ contribution, fluence was the most highly contributing factor towards channel width, as can be seen from figure 5a. The channel width increased with the angle of tilt (see figure 4e), quite like an ideal case, and the angle had a $24 \%$ contribution to the channel width. There is a little variation observed in channel width with spot overlap (see figure $4 f$ ), reiterating the fact that excessive lateral ablation rendered the extent of spot overlap inconsequential. The maximum edge roughness and channel width obtained with the chosen machining parameters were almost $8 \mu \mathrm{m}$ (at $0.9 \mathrm{~J} / \mathrm{cm}^{2}, 36^{\circ}$ and $20 \%$ spot overlap) and $190 \mu \mathrm{m}$ (at $1.3 \mathrm{~J} / \mathrm{cm}^{2}, 23^{\circ}$ and $40 \%$ spot overlap) respectively.

Machining, after coating metal films with a polymer film, was carried out. Figure 6 shows optical micrographs of some of the channels machined in, both, plain and PVA coated aluminum films. It is observed, upon comparing figure $6 \mathrm{a}$ with $6 \mathrm{~b}$ and $6 \mathrm{c}$ with $6 \mathrm{~d}$, that machining post poylmer coating caused a significant improvement in ablation quality. Channels machined in polymer coated film 


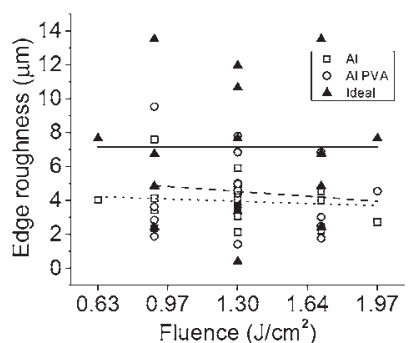

(a)

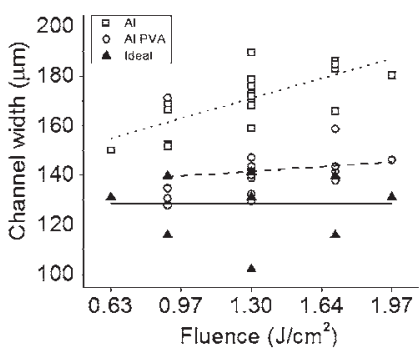

(d)

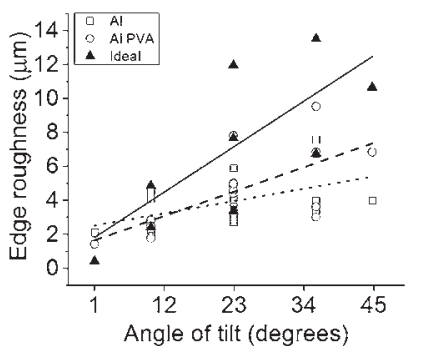

(b)

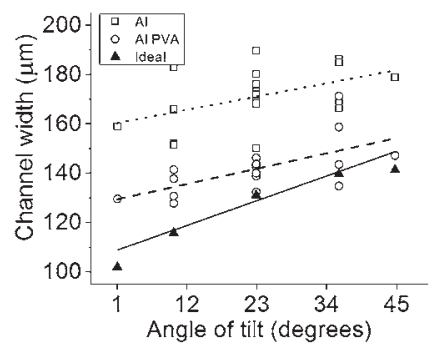

(e)

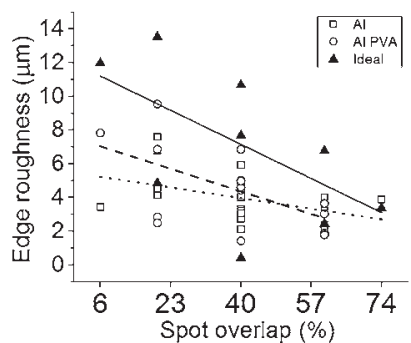

(c)

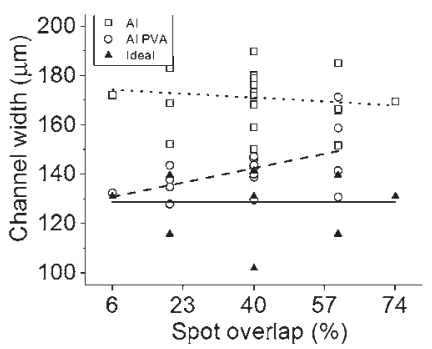

(f)

Figure 4. Plot showing variation of edge roughness and channel width (of channels machined with a $100 \mu \mathrm{m}$ wide laser spot) with fluence, angle and overlap, for uncoated and PVA coated aluminum. The lines are a guide to the eye only. Dotted line is for uncoated metal film, dashed line is for polymer coated metal film, whereas solid line represents the ideal variation.

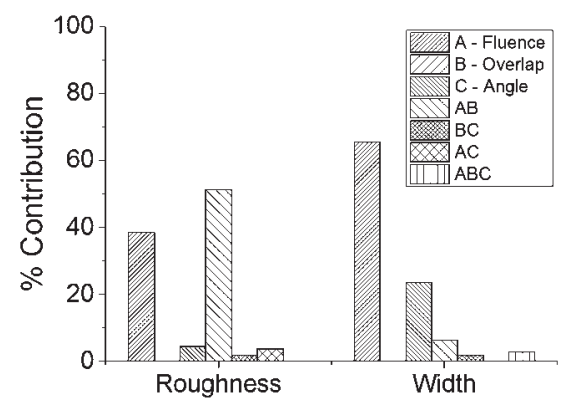

(a)

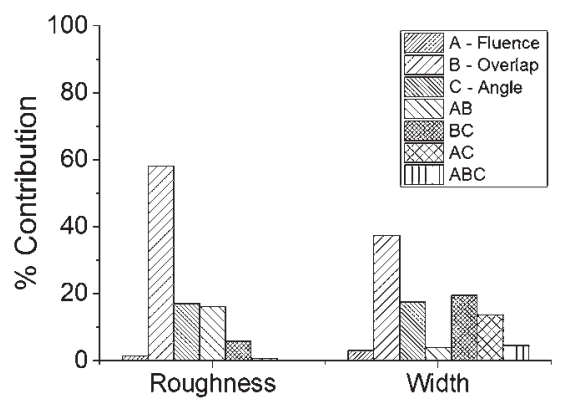

(b)

Figure 5. Contribution of various factors to edge roughness and channel width of oblique channels machined in (a) Plain aluminum film and (b) PVA coated aluminum film.

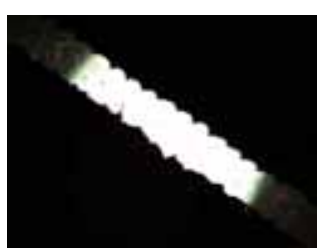

(a)

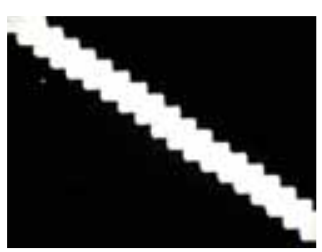

(b)

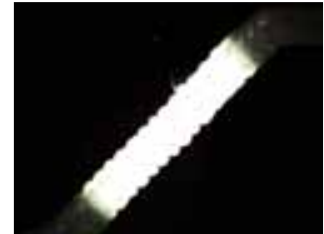

(c)

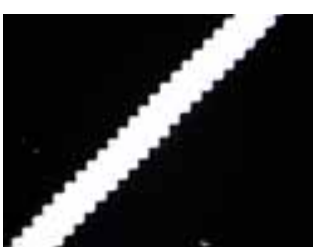

(d)

Figure 6. Optical micrographs of some oblique channels, machined with a $100 \mu \mathrm{m}$ wide laser spot, in aluminum, without (a and c) and with polymer coating ( $\mathrm{b}$ and $\mathrm{d}$ ). Channels a and $\mathrm{b}$ are machined at $0.9 \mathrm{~J} / \mathrm{cm}^{2}, 36^{\circ}$ and $20 \%$ spot overlap. Channels $\mathrm{c}$ and $\mathrm{d}$ are machined at $1.3 \mathrm{~J} / \mathrm{cm}^{2}, 44.9^{\circ}$ and $40 \%$ spot overlap. (a) On plain aluminum, (b) On PVA coated aluminum, (c) On plain aluminum, and (d) On PVA coated aluminum. 
had a sharply defined channel boundary, that closely resembled the series of laser spots falling one after the other, like an ideal channel as shown in figure 3 . The variation in roughness and width of channels machined in polymer coated aluminum film with fluence, angle of tilt and spot overlap was similar to that of those machined in plain aluminum film. However, since the extent of lateral overcut was smaller in case of polymer coated aluminum film, the overlap played a much more significant role, with $58 \%$ contribution, in determining the edge roughness and the channel width (refer figure 5b). This is unlike the case of plain aluminum film where the fluence was the most significant factor, with $60 \%$ contribution, owing to high lateral overcut (figure 5a).

The channel width was affected most by the spot overlap, with $37 \%$ contribution, and then by the angle of tilt and the angle-spot overlap interaction, with their contributions close to $20 \%$. It is to be noted, however, that the edge roughness of the oblique channels machined in polymer coated aluminum film was always higher, and the channel width, lower, compared to that of channels machined in plain aluminum film. This is similar to the case of ideal channels, which had the highest roughness and the lowest width of the three. The maximum edge roughness and channel width obtained in the case of PVA coated aluminum film with the chosen machining parameters were 9.5 $\mu \mathrm{m}$ (at $0.9 \mathrm{~J} / \mathrm{cm}^{2}, 36^{\circ}$ and $20 \%$ spot overlap) and $171 \mu \mathrm{m}$ (at $0.9 \mathrm{~J} / \mathrm{cm}^{2}, 36^{\circ}$ and $60 \%$ spot overlap) respectively.

The improvement in feature quality cannot be attributed to the interaction of the polymer layer with the laser pulse. This is because the $1 \mu \mathrm{m}$ thick polymer layer gets ablated within a few nanoseconds of the beginning of the pulse [12]. It is the action of the polymer layer as a clamping force that prevents the non-irradiated surrounding portions of the aluminum film from tearing and getting ejected at the slightest pressure buildup at the metal-glass interface. Indirectly, the polymer layer keeps the aluminum film adhered to the glass surface. Strength of adhesion to the substrate, hence, is an important factor in high resolution patterning of metal films.

\subsection{Chromium film machining (without and with polymer coating)}

Chromium films [chromium being a refractory metal [12]], undergo cracking during irradiation with excimer lasers [18]. Cracking occurs irrespective of the film getting ablated or not. The cracks get smaller as the fluence is increased. The fine cracking at higher fluence allows finer pieces of chromium to be ejected from the boundary (as well as the interior), allowing cleaner ablation.

The plots of edge roughness and channel width versus fluence, spot overlap and angle of tilt, for channels machined with a $100 \mu \mathrm{m}$ wide laser spot on chromium film on glass, both with and without polymer coating are shown in figure 7 . The edge roughness was found to remain independent of fluence, increase with angle and decrease with overlap for the case of plain chromium film only. With $53 \%$ contribution, fluence-spot overlap interaction contributed most to the edge roughness, followed by the anglespot overlap interaction, with $22 \%$ contribution. The

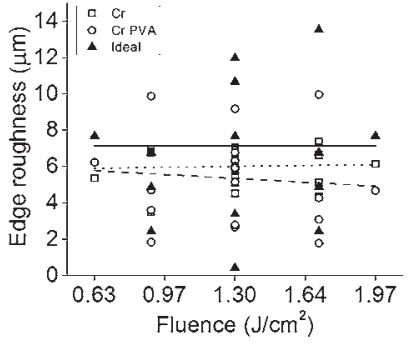

(a)

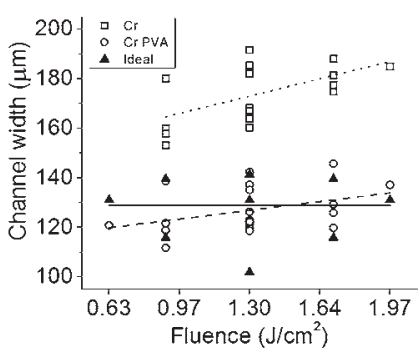

(d)

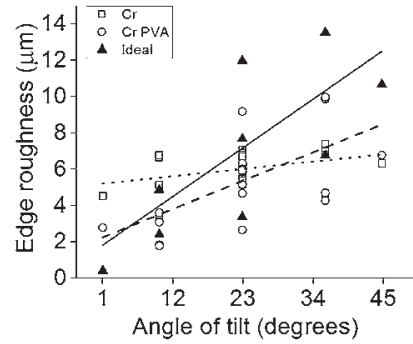

(b)

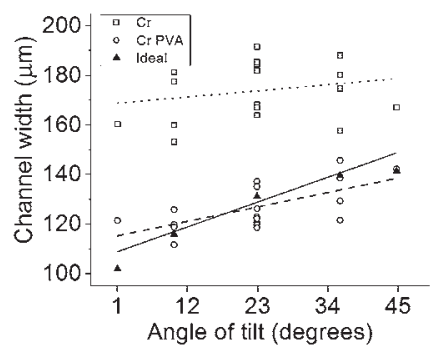

(e)

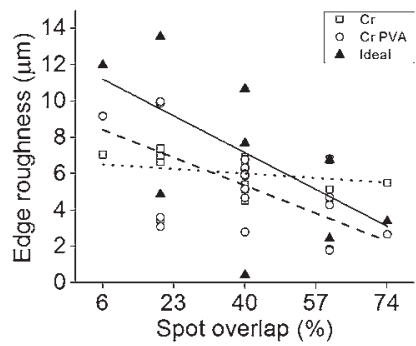

(c)

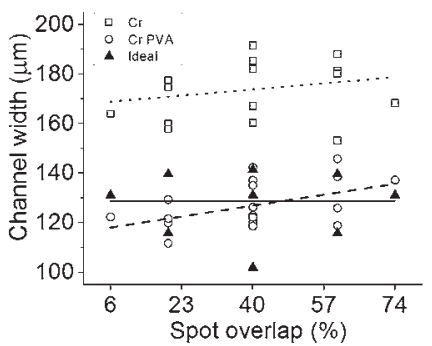

(f)

Figure 7. Plot showing variation of edge roughness and channel width (of channels machined with a $100 \mu \mathrm{m}$ wide laser spot) with fluence, angle and overlap, for uncoated and PVA coated chromium films. The lines are a guide to the eye only. Dotted line is for uncoated metal film, dashed line is for polymer coated metal film, whereas solid line represents the ideal variation. 
channel width was found to increase with fluence and angle of tilt, with fluence as the most significant parameter with 75\% contribution. All responses, except one, were similar to those of plain aluminum film. The width increased slightly with overlap, unlike the case of plain aluminum film. It was, in fact, similar to the case of PVA coated aluminum film. The reason for this is the absence of excessive lateral ablation due to good adherence of chromium film to glass and fine cracking upon irradiation. Larger ejection pressure is required to eject out the nonirradiated film when adherence to the substrate is high. Additionally, lesser ejection pressure is built up in this case because of the high volume specific heat of chromium that causes a slower rise in temperature as compared to that in aluminum. Fluence and overlap, interactively, were significant contributors to roughness (refer figure 8a). Fluence and angle contributed most towards the channel width. The maximum edge roughness and channel width obtained in the case of chromium film with the chosen machining parameters were $7.4 \mu \mathrm{m}$ (at $1.7 \mathrm{~J} / \mathrm{cm}^{2}, 36^{\circ}$ and $20 \%$ spot overlap) and $192 \mu \mathrm{m}$ (at $1.3 \mathrm{~J} / \mathrm{cm}^{2}, 23^{\circ}$ and $40 \%$ spot overlap) respectively.

Optical micrographs of some of the oblique channels machined in, both, plain and PVA coated chromium films are shown in figure 9. Similar to the observation for aluminum films, micromachining post polymer coating gave a significant improvement in feature quality. Features in PVA coated metal film were sharply machined with enhanced edge quality. The variations in edge roughness and channel width as functions of the machining parameters were similar to those of polymer coated aluminum films.

In the case of polymer coated aluminum film, the spot overlap contributed most significantly to the edge roughness and channel width, not only as an independent factor, but also in interaction with other factors. This was due to the fact that the aluminum film is prone to excessive lateral ablation, and the effect of angle of tilt (or spot overlap) was evident only at low fluence. In case of polymer coated chromium film, angle of tilt was, independently, the most significant factor, with $59 \%$ contribution, and the spot overlap came second, with $31 \%$ contribution. This was much closer to the response of an ideal channel, and is attributed to the high adherence of chromium to the substrate, and the clamping action of the polymer coating. The channel width, like the former, was always lower for polymer coated chromium film compared to that of plain chromium film. It was affected most by the angle of tilt, with 54\% contribution, followed by spot overlap, with $24 \%$ contribution. The maximum edge roughness and channel width obtained in the case of PVA coated chromium film with the chosen machining parameters were $10.0 \mu \mathrm{m}$ (at $1.7 \mathrm{~J} / \mathrm{cm}^{2}, 36^{\circ}$ and $20 \%$ spot overlap) and $146 \mu \mathrm{m}$ (at 1.7 $\mathrm{J} / \mathrm{cm}^{2}, 36^{\circ}$ and $60 \%$ spot overlap) respectively.

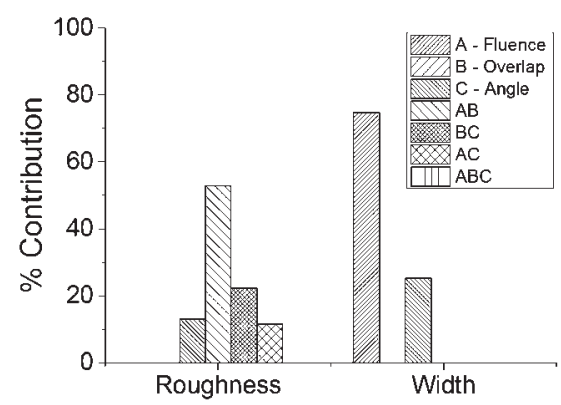

(a)

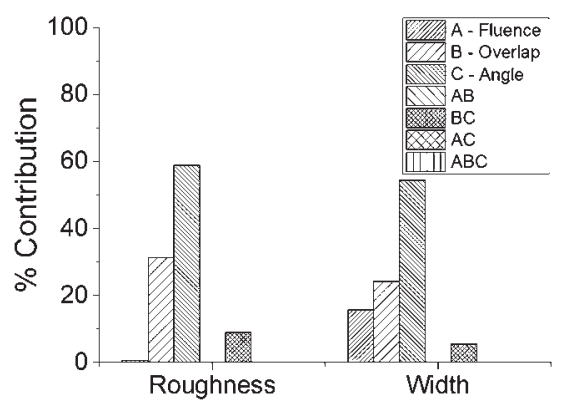

(b)

Figure 8. Contribution of various factors to edge roughness and channel width of oblique channels machined in (a) Plain chromium film and (b) PVA coated chromium film.

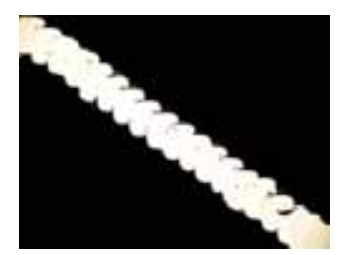

(a)

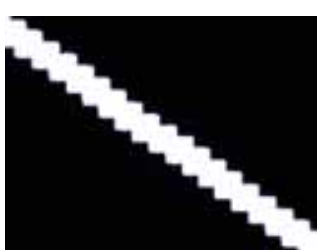

(b)

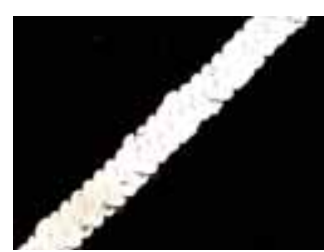

(c)

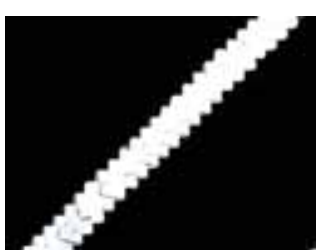

(d)

Figure 9. Optical micrographs of some oblique channels, machined with a $100 \mu \mathrm{m}$ wide laser spot, in chromium, without (a and c) and with polymer coating ( $\mathrm{b}$ and $\mathrm{d}$ ). Channels a and $\mathrm{b}$ are machined at $0.9 \mathrm{~J} / \mathrm{cm}^{2}, 36^{\circ}$ and $20 \%$ spot overlap. Channels $\mathrm{c}$ and $\mathrm{d}$ are machined at $1.3 \mathrm{~J} / \mathrm{cm}^{2}, 44.9^{\circ}$ and $40 \%$ spot overlap. (a) On plain chromium, (b) On PVA coated chromium, (c) On plain chromium, and (d) On PVA coated chromium. 


\section{Conclusions}

A set of machining experiments were carried out on uncoated and polymer-coated aluminum and chromium films, coated on a glass substrate. Oblique channels, machined with 100 $\mu \mathrm{m}$ wide laser spots, have been fabricated by excimer laser micro machining using a square laser spot. The edge roughness and width of ideal oblique microchannels were not found to be affected by fluence, while both these quantities increased with the angle of tilt. Only the edge roughness decreased with increase in spot overlap, while the channel width was not affected. A significant improvement in ablation quality, with sharper and cleaner features, were observed while machining post coating the metal films with the polymer layer. The effects of the machining parameters on the machined channels are summarized below, first for the edge roughness and then for the channel width.

\subsection{Effect on edge roughness}

Plain aluminum film - In the case of plain aluminum films, fluence was found to affect the edge roughness both as an independent factor (with $38 \%$ contribution) and in interaction with the spot overlap (with $51 \%$ contribution), albeit in opposite senses. Hence, overall, the edge roughness was found to be little affected by the fluence for plain aluminum films, very unlike the case of straight channels. The edge roughness decreased with spot overlap, and was mostly affected by the spot overlap at small fluences. It increased with the angle of tilt, and was comparatively weakly affected by the angle. The maximum edge roughness of 8 $\mu \mathrm{m}$ was observed.

PVA coated aluminum film - The variations in the edge roughness were similar to those of plain aluminum films. However, the edge roughness was most affected by the spot overlap (with $58 \%$ contribution). Angle of tilt and fluencespot overlap interaction, both, have about $16 \%$ contributions to the edge roughness. The maximum edge roughness of $9.5 \mu \mathrm{m}$ was observed in this case.

Plain chromium film - Chromium films were observed to ablate more cleanly compared to aluminum films, owing to the strong adhesion of chromium films to the glass substrate. As a result, the roughness of channels machined in chromium was more than that in case of aluminum films, an observation contrary to the case of machining straight channels. The edge roughness, in case of plain chromium film, had variations similar to that in case of plain aluminum films. However, it was not affected independently either by fluence or by spot overlap. With $53 \%$ contribution, fluence-spot overlap interaction contributed most to the edge roughness, followed by the angle-spot overlap interaction, at $22 \%$ contribution. The maximum edge roughness of $7.4 \mu \mathrm{m}$ was observed in this case.

PVA coated chromium film - The variation in edge roughness was similar to that in case of PVA coated aluminum films. The edge roughness was affected mostly by the angle of tilt and the spot overlap, with $59 \%$ and $31 \%$ contributions, respectively. The maximum edge roughness of $10.0 \mu \mathrm{m}$ was observed in this case.

In case of plain metal films, the interaction of fluence and spot overlap most significantly affected the edge roughness, whereas spot overlap and angle of tilt were the most significant factors in determining edge roughness for polymer coated metal films. (It is relevant to mention here that spot overlap did not affect the edge roughness of straight channels machined in polymer coated metal films.) In this regard, polymer coated metal films underwent close to ideal ablation, little affected by fluence. Hence, polymer coating created a clamping force preventing large pieces of non-irradiated metal film from getting ejected, leading to a sharp ablation and improved feature quality.

\subsection{Effect on channel width}

Plain aluminum film - The channel width was affected most by the fluence ( $65 \%$ contribution), followed by the angle of tilt ( $24 \%$ contribution). The channel width was not found to be affected by spot overlap for plain aluminum films. The maximum channel width of $190 \mu \mathrm{m}$ was observed.

PVA coated aluminum film - The channel width was affected most by the spot overlap (with $37 \%$ contribution), and then by the angle of tilt and the angle-spot overlap interaction, with their contributions close to $20 \%$. The maximum channel width of $171 \mu \mathrm{m}$ was observed in this case.

Plain chromium film - The channel width was found to increase with fluence and angle of tilt, with fluence as the most significant parameter with $75 \%$ contribution. The maximum channel width of $192 \mu \mathrm{m}$ was observed in this case.

PVA coated chromium film - The variation in channel width was similar to that in case of PVA coated aluminum films. The channel width was affected by these parameters, with $54 \%$ and $24 \%$ contributions. The maximum channel width of $146 \mu \mathrm{m}$ was observed in this case.

The channel widths, in all cases, were observed to increase with fluence and angle of tilt. Spot overlap had a little effect on channel width. High fluence causes excessive lateral ablation, thereby increasing the channel width. The extent of overcut, however, was less in polymer coated metal films compared to that in plain metal films. Because of sharp and close to ideal ablation in polymer coated metal films, the widths of channels machined in such films were always lower compared to those in case of plain metal films.

A novel technique of machining sharp curved features on thin metal films using a square laser spot has been demonstrated. This technique helps in curtailing the edge roughness of the microfeatures to values less than $10 \mu \mathrm{m}$, when the highest value of the ideally expected roughness is 
close to $13 \mu \mathrm{m}$, at $20 \%$ spot overlap and $36^{\circ}$ angle of tilt. Channel width has been demonstrated to be reduced to 150 $\mu \mathrm{m}$ from $190 \mu \mathrm{m}$ by using a polymer coating. Ideally expected channel width is about $140 \mu \mathrm{m}$, which is close to the size of the square laser spot. The developed fabrication technique can be an alternative approach for the direct machining of the metal films for mass production of the MEMS, microwave and photonics devices, micro fluidic channels, etc.

\section{Acknowledgments}

The authors thank the Department of Science and Technology, Government of India (FIST DST Project No. 20060268), and the Indian Space Research Organization (Project No. 20120040) for partial support.

\section{References}

[1] Maier S A 2007 Plasmonics: Fundamentals and applications. Springer, New York

[2] Nagpal P, Lindquist N C, Oh S-H and Norris D J 2009 Ultrasmooth patterned metals for plasmonics and metamaterials. Science 325(5940): 594-597

[3] Ramakrishna S A and Grzegorczyk T M 2009 Physics and applications of negative refractive index materials, vol. 186. CRC Press, Boca Raton, Florida

[4] Herzig H P 1997 Micro-optics: Elements, systems and applications. CRC Press

[5] Watts C M, Liu X and Padilla W J 2012 Metamaterial electromagnetic wave absorbers. Adv. Mater. 24(23): OP98OP120

[6] Weiner J 2009 The physics of light transmission through subwavelength apertures and aperture arrays. Reports Progr. Phys. 72(6): 064401

[7] Dhote A M, Shreekala R, Patil S I, Ogale S B, Venkatesan T and Williams C M 1995 Pulsed excimer laser etching of
$L a_{0.75} \mathrm{Ca}_{0.25} \mathrm{MnO}_{X}$ thin films. Appl. Phys. Lett. 67(24): 3644-3646

[8] Andrew J E, Dyer P E, Greenough R D and Key P 1983 Metal film removal and patterning using a $\mathrm{XeCl}$ laser. Appl. Phys. Lett. 43(11): 1076-1078

[9] Toth Z, Hopp B, Kantor Z, Ignacz F, Szörényi T and Bor Z 1995 Dynamics of excimer laser ablation of thin tungsten films monitored by ultrafast photography. Appl. Phys. A 60(5): 431-436

[10] Miyamoto K and Hayashi $\mathrm{H} 1995$ Process of thin $\mathrm{Cu}$ film removal by $\mathrm{KrF}$ excimer laser. Rev. Laser Eng. 23(12): 1081-1089

[11] Benjamin P and Weaver C 1961 The adhesion of evaporated metal films on glass. Proc. R. Soc. Lond. A, Math. Phys. Sci. 261(1307): 516-531

[12] Akhtar S N, Sharma S, Dayal G, Ramakrishna S A and Ramkumar J 2015 Microfeature edge quality enhancement in excimer laser micromachining of metal films by coating with a sacrificial polymer layer. J. Micromech. Microeng. 25: 065001

[13] Dayal G, Akhtar S N, Ramakrishna S A and Ramkumar J 2013 Excimer laser micromachining using binary mask projection for large area patterning with single micrometer features. ASME J. Micro Nano Manuf. 1(3): 031002-1-7

[14] Montgomery D C 2008 Design and analysis of experiments. John Wiley \& Sons.

[15] Akhtar S N, Ramakrishna S A and Ramkumar J 2013 Microfeature edge quality optimization in excimer laser ablation of metallic film. In: Proceedings of the ASME 2013 International Mechanical Engineering Congress \& Exposition, San Diego, California, USA

[16] Matthias E, Reichling M, Siegel J, Käding O W, Petzoldt S, Skurk H, Bizenberger P and Neske E 1994 The influence of thermal diffusion on laser ablation of metal films. Appl. Phys. A 58(2): 129-136

[17] Jensen M 2004 Laser micromachining of polymers. $\mathrm{PhD}$ thesis, Technical University of Denmark, Denmark

[18] Matthias E, Siegel J, Petzoldt S, Reichling M, Skurk H, Käding O and Neske E 1995 In-situ investigation of laser ablation of thin films. Thin Solid Films 254(1): 139-146 